\title{
Silver-related local centres in cadmium sulfide
}

\author{
L.V. Borkovskaya, B.M. Bulakh, L.Yu. Khomenkova, I.V. Markevich \\ Institute of Semiconductor Physics, NAS Ukraine, 45 prospect Nauki, Kyiv, 03028, Ukraine, \\ Tel: (044) 265-72-34,Fax: (044)265-83-44,E-mail: bork@lumin.semicond.kiev.ua
}

\begin{abstract}
Silver-related defects and their diffusion in CdS crystals were investigated. The impurity was introduced in the crystal and extracted from it under electric field $E_{d}=10^{2} \mathrm{~V} / \mathrm{cm}$ at $T=300-450^{\circ} \mathrm{C}$ both parallel and perpendicular to the $c$-axis. The only local centre that was proved to appear after Ag introduction and to disappear after its extraction was deep acceptor responsible for emission band $\lambda_{m}=610 \mathrm{~nm}$. Photo-enhanced defect reaction resulting in photosensitivity degradation was shown to occur after Ag incorporation. It was found that diffusion anisotropy took place, Ag diffusion being some times faster parallel to the $c$-axis.
\end{abstract}

Keywords: local centres, diffusion.

Paper received 07.06.01; revised manuscript received 18.06.01; accepted for publication 13.07.01.

\section{Introduction}

To get information about impurity-related local centres in crystal lattice of any semiconductor an impurity is introduced in it and then characteristics of doped material and undoped one are compared. As a rule, doping is carried out under growing or by diffusion from the surface at rather high temperatures. Under these conditions creation of native defects and their complexes is highly probable, the types of these defects and their densities depending on temperature, ambient atmosphere and type of impurity [1]. The presence of such «outside» 11 defects makes difficulties for identification of defects related to introduced impurity. At the same time, it is known that diffusion of some impurities, particularly, copper and silver can be accelerated in many semiconductors, including CdS, by external electric field [2-4]. In this case application of electric field allows to lower the temperature of the doping process and so to reduce the probability of outside defect creation. Besides, one can introduce the impurity in the sample and then extract that from it changing the polarity of the electric field [4]. These procedures can be made over and over again, which facilitates the identification of the impurity-related centres. In the present work Ag-related local centres in CdS crystals were investigated by this technique.

\section{Experimental procedure and results}

Nominally undoped high-resistivity $\left(\rho>10^{8} \Omega \cdot \mathrm{cm}\right)$ bulk and platelet $\mathrm{CdS}$ crystals were used. Initial crystals have bright yellow colour. The impurity was introduced in the sample from electrode containing Ag, the other electrode being pure In. The sample was fastened horizontally on the heater, heated to the temperature $T_{d}$ from $300-450{ }^{\circ} \mathrm{C}$ range and then direct electric field $E_{d}=100-300 \mathrm{~V} / \mathrm{cm}$ was applied to it so that the electrode containing $\mathrm{Ag}$ was the anode [3]. After time interval $\Delta t=5 \ldots 45 \mathrm{~min}$ the electric field was switched off and the sample was cooled to room temperature. Similar procedure was also made with some CdS:Ag crystals doped under growing, both electrodes being pure In. These crystals have bright brown colour.

Soon after the electric field was switched on, in initially undoped crystals a dark region appeared near the anode and then spread to the cathode. After cooling to room temperature the dark region acquired bright brown colour like CdS:Ag crystals while the rest sample remained bright yellow. When the sample was heated again to $T_{d}$ and electric field of opposite direction was applied to it, the brown region shortened with time and the yellow one spread to the new cathode. In previously doped crystals after electric field switching on a light region appeared 

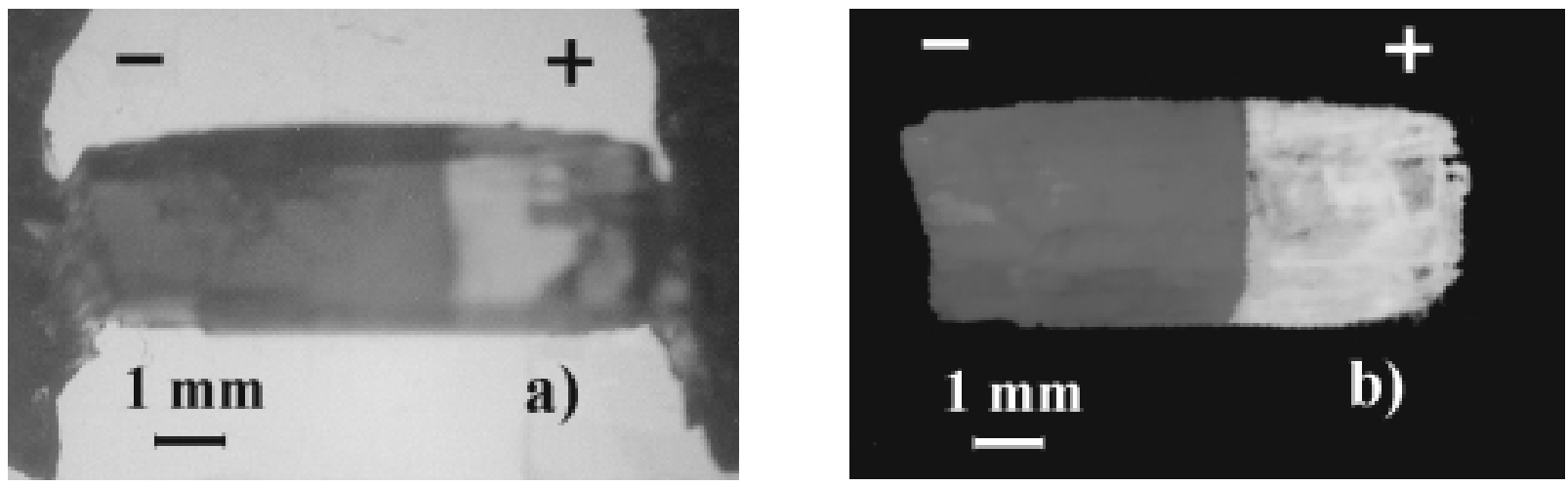

Fig. 1. a) CdS:Ag crystal partially purified at $400^{\circ} \mathrm{C}\left(E_{D}=200 \mathrm{~V} / \mathrm{cm}^{2}, \Delta t_{D}=30 \mathrm{~min}\right)$ under visible light at $300 \mathrm{~K}$.

b) CdS:Ag crystal partially purified at $400^{\circ} \mathrm{C}\left(E_{D}=200 \mathrm{~V} / \mathrm{cm}^{2}, \mathrm{D} t=30 \mathrm{~min}\right)$ under excitation with $\lambda_{\mathrm{exc}}=365 \mathrm{~nm}$ mercury lamp line at $T=77 \mathrm{~K}$.

near the anode and spread with time along the sample until only a narrow dark strip remained near the cathode. After cooling to room temperature the light region became bright yellow like undoped crystal. So, one can suppose that coloration of initially undoped crystal under electric field application is caused by Ag incorporation while bleaching of CdS:Ag crystal under electric field application id due to Ag extraction. The bleached crystal can be made bright brown again by application of opposite direction electric field. Thus, incorporation of $\mathrm{Ag}$ in any region of the sample and extraction of this impurity from it could be made over and over again. A sharp boundary was found to form between brown (doped with $\mathrm{Ag}$ ) and yellow (undoped or purified) sample regions (Fig. 1, a). Transmission spectra measurements show that after Ag incorporation transparency reduced and absorption edge shifted to the long wavelength side (Fig. 2). After Ag extraction transmission spectra of undoped crystal restored.

\subsection{Energy levels of Ag-related centres}

Photoluminescence (PL), photocurrent (PC) and thermally stimulated current (TSC) spectra of the same crystal region were measured before and after Ag incorporation and then after its extraction. For electrical measurements additional In electrodes were applied.

The difference in PL spectra of doped and undoped regions was well conspicuous under illumination with $365 \mathrm{~nm}$ line of mercury lamp at $77 \mathrm{~K}$ : the undoped region was bright green, while the doped one emitted orange light. The boundary between green and orange parts exactly coincided with that between yellow and brown regions (Fig.1, b).

PL spectra of initially undoped and doped CdS crystals measured before and after Ag incorporation or extraction are shown in Fig. 3 (a, b). It is seen that incorporation of $\mathrm{Ag}$ results in appearance of orange band at $\lambda_{m}=610 \mathrm{~nm}$ and quenching of edge green emission. The latter can be caused by re-absorption of the edge emis- sion in the crystal due to the shift of absorption edge (Fig.2). After Ag extraction orange emission disappears and green band restores. The intensity of red band at $\lambda_{m}=715 \mathrm{~nm}$ that was also present in PL spectra of both doped and undoped sample regions was found to be factually independent on $\mathrm{Ag}$ incorporation or extraction. In some undoped crystals so-called self-activated orange emission at $\lambda_{m}=590 \mathrm{~nm}$ was observed in addition to green and red emissions. These crystals were supplied with two In electrodes and PL spectra were measured near the same electrode in the initial state and after electric field application at $T_{d}=400{ }^{\circ} \mathrm{C}$ during $\Delta t=30 \mathrm{~min}$ so that this electrode was first the anode and then the cathode. Any influence of the electric field on PL spectra was not found in this case.

Incorporation of $\mathrm{Ag}$ was accompanied with the increase of crystal photosensitivity in the whole wavelength range, the most increase being observed in extrinsic «or-

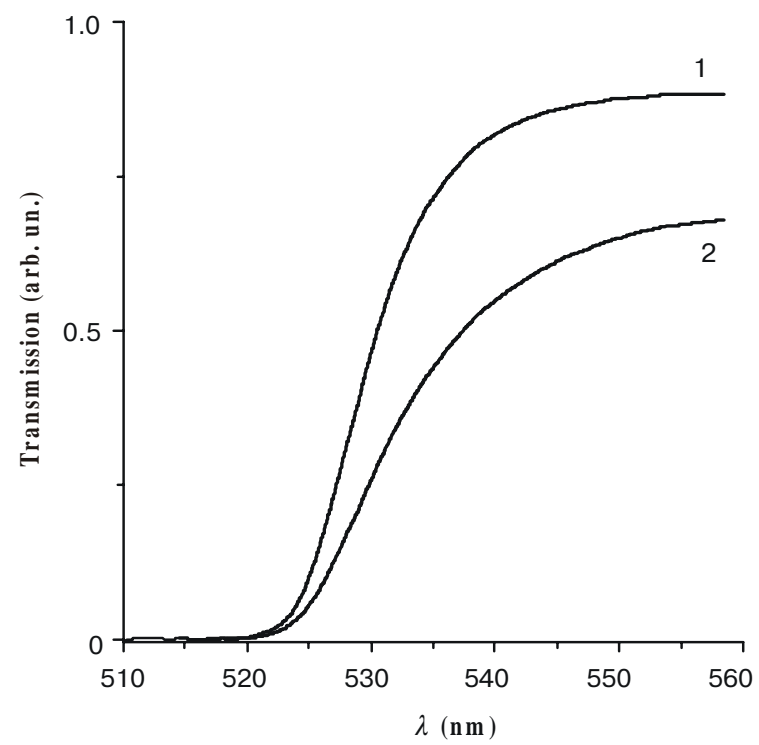

Fig. 2. Transmission spectra of CdS crystal before (1) and after (2) Ag incorporation and after next Ag extraction (1). 


\section{L.V. Borkovskaya et al.: Silver-related local centres in cadmium sulfide}
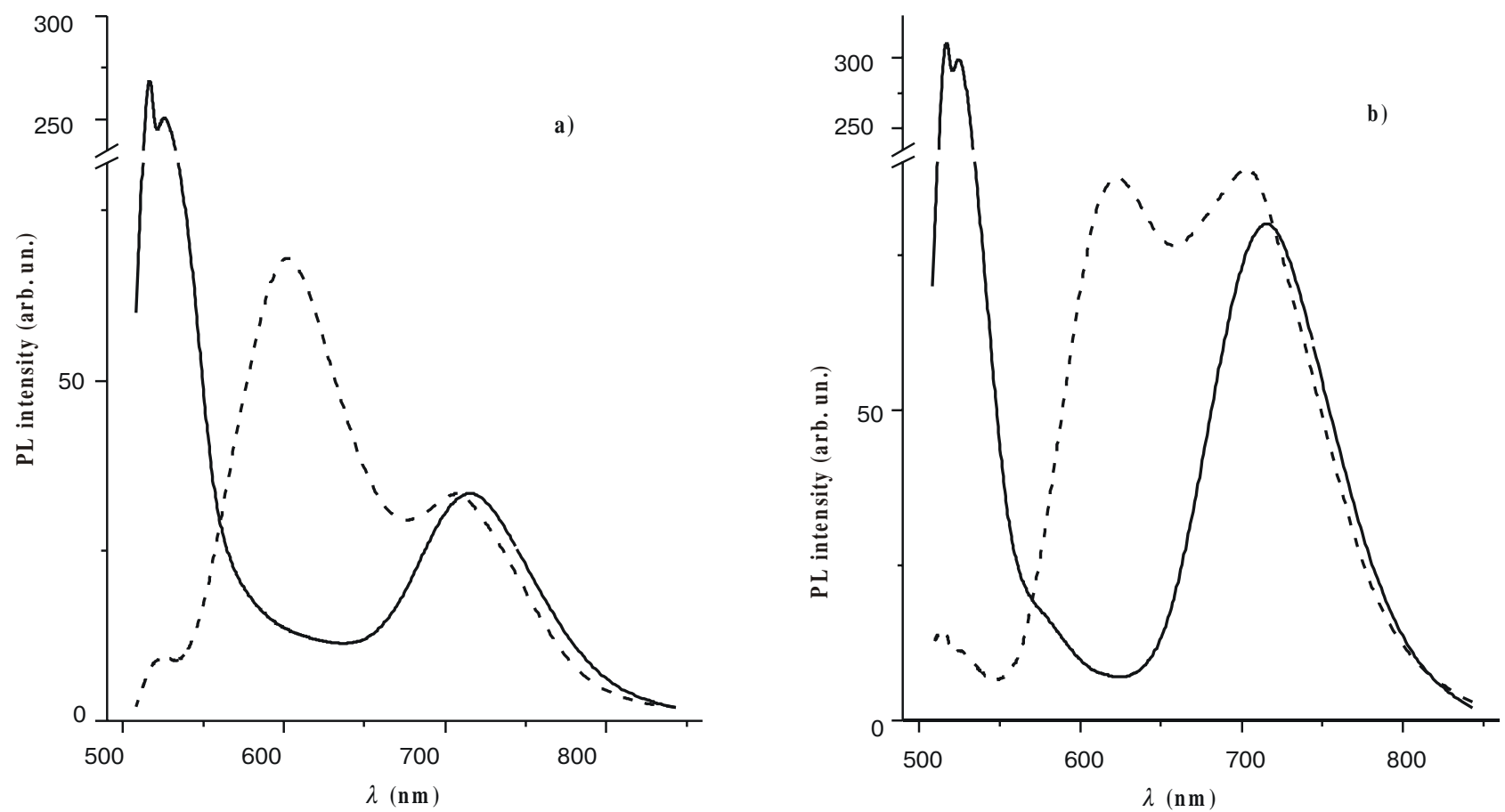

Fig. 3. Photoluminescence spectra of CdS crystals after Ag incorporation (dashed line) and Ag extraction (solid line): (a) nominally undoped crystal; (b) - crystal doped with Ag during growth.

ange» region (Fig. 4). In order to elucidate whether photoenhanced defect reactions took place in investigated crystals PC spectra of undoped, then doped and next purified sample region were measured at $300 \mathrm{~K}$ after cooling from 450 to $300 \mathrm{~K}$ first in dark and next under visible light illumination [5]. In undoped sample a slight photosensitizing reaction was observed (Fig. 4, curves 1,2). Such reaction often takes place in nominally undoped $\mathrm{CdS}$ and has been shown to be due to rearrangement of native defects [5]. After Ag incorporation considerable photosensitivity degradation was observed as a result of illumination in 450-30 $\mathrm{K}$ temperature range (Fig4, curves 3,4). Similar reaction had been earlier investigated in $\mathrm{CdS}: \mathrm{Cu}$ crystals [5]. In $\mathrm{CdS}$ doped with $\mathrm{Ag}$ degradation reaction was observed for the first time and was found to occur at higher temperatures then in $\mathrm{CdS}: \mathrm{Cu}$. When $\mathrm{Ag}$ was extracted from the sample, degradation reaction disappeared and photosensitizing reaction restored.

In Fig. 5 TSC spectra of CdS:Ag crystal before and after Ag extraction were shown. It is seen that none TSC peak disappeared when Ag was extracted. The decrease of current level in whole temperature range after sample purification is obviously due to the decrease of photosensitivity (Fig. 4). In TSC spectra of the most initially undoped crystals only peak at $T_{m}=95 \mathrm{~K}$ was present and any new peak did not appear after Ag incorporation.

\subsection{Diffusion characteristics}

Diffusion of Ag under electric field was carried out both parallel and perpendicular to the c-axis. Corresponding diffusion coefficients $D_{\|}$and $D_{\perp}$ were calculated from Einstein's relation $D=\mu k T_{d} l e$, where $\mu=l_{d} / E_{d} \Delta t_{d}-$ impurity ion mobility, $l_{d}$ - length of doped (or purified) region, $k$ - Boltzmann constant, $e$ - electron charge. Activation energies of diffusion $E_{d \| \mid}$ parallel and $E_{d \perp}$ per-

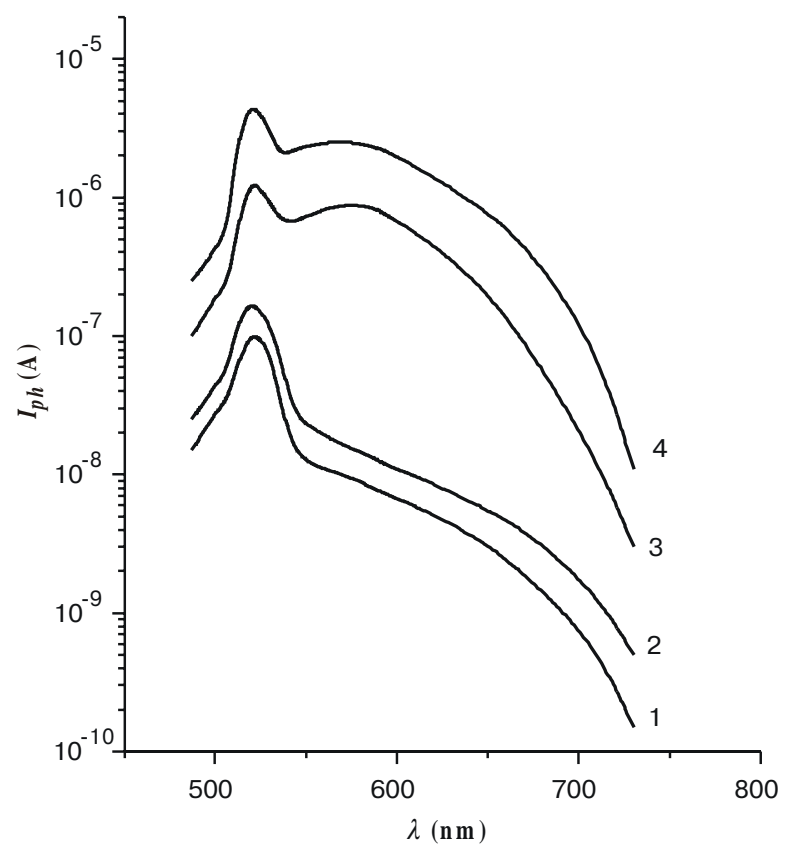

Fig. 4. Photocurrent spectra of CdS crystal before $(1,2)$ and after $(3,4) \mathrm{Ag}$ incorporation after cooling from $420 \mathrm{~K}$ to $300 \mathrm{~K}$ in dark $(1,4)$ and under intrinsic light illumination $(2,3)$. 


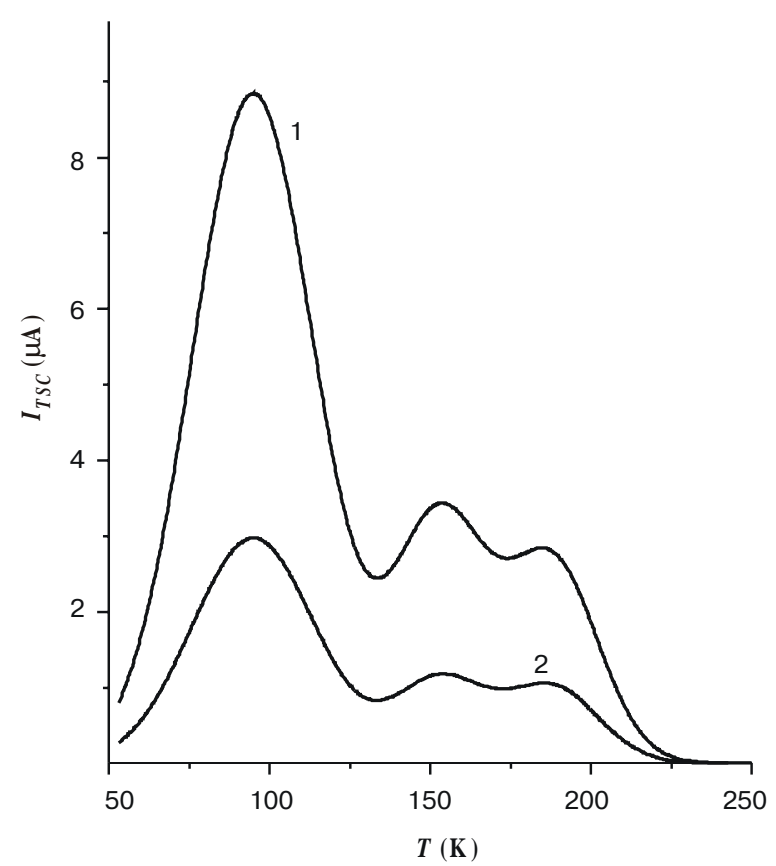

Fig. 5. TSC spectra of CdS:Ag crystal before (1) and after (2) Ag extraction.

pendicular to the $c$-axis were determined from $D_{\|}$and $D_{\perp}$ temperature dependencies (Fig. 6). Diffusion parameters obtained from the velocities of both Ag incorporation and extraction processes were proved to coincide. As it is seen from fig.6, considerable diffusion anisotropy takes place, diffusion being some times faster parallel to the $c$-axis. The values of $E_{d \| \mid}$ and $E_{\perp}$ are different and equal to $1.1 \pm 0.05$ and $1.35 \pm 0.05 \mathrm{eV}$ correspondingly.

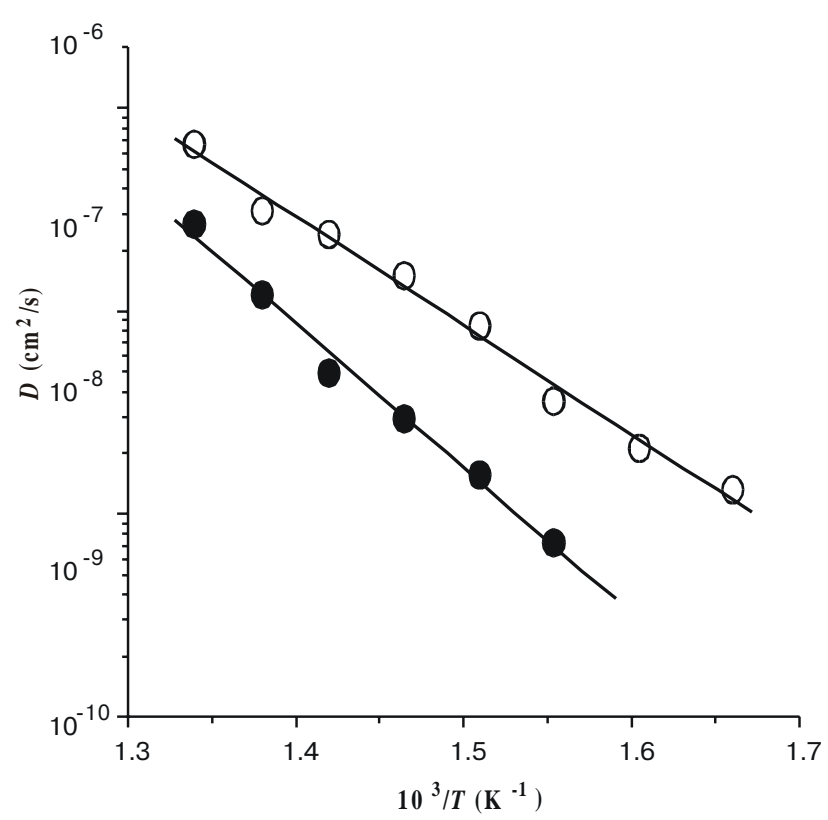

Fig.6. Temperature dependencies of Ag coefficients of diffusion parallel (1) and perpendicular (2) to the $c$-axis.

\section{Discussion}

The basic Ag-related defects in CdS are silver in the $\mathrm{Cd}$ site $\mathrm{Ag}_{\mathrm{Cd}}$ and silver interstitial $\mathrm{Ag}_{i}$. These defects must act as a single charged acceptor and a single charged donor correspondingly [6]. Appearance of PL band at $\lambda_{m}=610 \mathrm{~nm}$ after Ag incorporation and its quenching after $\mathrm{Ag}$ extraction prove that this band is due to $\mathrm{Ag}$ related defects. It is known that a number of orange emission bands can be observed in CdS PL spectra in 580$640 \mathrm{~nm}$ wavelength range. Some of these bands are ascribed to different impurities, others (so-called self-activated) are thought to be related to native defects $[6,7]$. All orange bands were shown to result from recombination of free electrons on complex centres consisting of deep acceptors and shallow donors [8]. One can conclude, therefore, that acceptor responsible for the band at $\lambda_{m}=610 \mathrm{~nm}$ is $\mathrm{Ag}_{\mathrm{Cd}}$. At the same time the orange band at $\lambda_{m}=590 \mathrm{~nm}$ which is observed in nominally undoped crystals and does not change after action of electric field is obviously due to immobile native acceptor $V_{\mathrm{Cd}}$.

In CdS crystals doped with $\mathrm{Ag}$, as a rule, intensive red emission at $\lambda_{m}=710-720 \mathrm{~nm}$ was also observed and usually ascribed to Ag-related centres [7,9,10]. On the other hand, such band is often present in PL spectra of undoped $\mathrm{CdS}$ (self-activated red band) [6,7]. An emission band at $\lambda_{m}=710 \mathrm{~nm}$ takes place in PL spectra of investigated crystals too. Our data show, however, that Ag incorporation or extraction does not influence on this band intensity in neither initially «pure», nor initially doped crystals. One can think, therefore, that this band is due to native defects. Perhaps, the presence of Ag enables the creation of defects responsible for self-activated red emission. Thus, by means of electric field it is possible to separate emission bands related to immobile native defects and mobile impurities.

The direction of drift in electric field shows that silver incorporates in crystal and diffuses in it as positively charged ions, i.e. interstitials $\mathrm{Ag}_{\mathrm{i}}{ }^{+}$, so, these defects are present in the crystal during its doping or purification. Then acceptors $\mathrm{Ag}_{\mathrm{Cd}}$ create due to reaction

$\mathrm{Ag}_{i}+V_{\mathrm{Cd}}=\mathrm{Ag}_{\mathrm{Cd}}$

In available literature $\mathrm{Ag}_{\mathrm{i}}$ centres were stated to be deep donors $[9,11]$ that in highly compensated material will act as deep electron traps. The analysis of TSC data shows, however, that any new electron traps do not appear after Ag incorporation or disappear after its extraction. The absence of $\mathrm{Ag}_{\mathrm{i}}$ centres in noticeable concentrations can be explained by the shift of the equilibrium (1) to the right side.

The results of investigation of Ag diffusion dependence on crystallographic direction turned out quite interesting. The available information about impurity diffusion anisotropy in II-VI compounds is rather scanty. For the first time this effect in $\mathrm{CdS}$ was reported for $\mathrm{Cu}$, ratio $D_{\mathrm{Cu} \perp} / D_{\mathrm{Cu}||}>10$ being found [12]. Then In [13] and Ga [14] diffusion anisotropy in CdS with $D_{\wedge}=3 D_{||}$was ob- 


\section{L.V. Borkovskaya et al.: Silver-related local centres in cadmium sulfide}

served. Later we investigated $\mathrm{Cu}$ diffusion in $\mathrm{CdS}$ under electric field both perpendicular and parallel to the caxis [15] and obtained data coincided with [12]. Dependence of Ag diffusion on crystallographic direction was not reported thus far. The fact that the direction of $\mathrm{Ag}$ diffusion anisotropy $\left(D_{\|}>D_{\perp}\right)$ is opposite to the direction of $\mathrm{Cu}$ diffusion anisotropy $\left(D_{\|}<D_{\perp}\right)$ is surprising. In general, different directions of diffusion anisotropy for different impurities were earlier found in hexagonal Zn crystals [16]. This effect was accounted for different interaction of impurity-related defects with $\mathrm{Zn}$ vacancies due to different charges of these defects in $\mathrm{Zn}$ crystal lattice [16]. However, both $\mathrm{Cu}$ and $\mathrm{Ag}$ diffuse in $\mathrm{CdS}$ interstitially as single charged ions $\mathrm{Cu}_{i}^{+}$and $\mathrm{Ag}_{i}{ }^{+}$, so the same diffusion mechanism could be expected for these impurities. At the same time, opposite directions of diffusion anisotropy indicate that mechanisms of copper and silver diffusion in CdS are different. To elucidate the nature of this phenomenon more detailed investigations are planned.

\section{Conclusions}

Diffusion of Ag in high-resistivity CdS crystals parallel and perpendicular to the $c$-axis was carried out. The impurity was introduced in the sample and extracted from it under electric field $E_{d}=50-300 \mathrm{~V} / \mathrm{cm}$ at $T_{d}=300-450 \mathrm{~K}$. It was shown that $\mathrm{Ag}$ incorporation (extraction) resulted in:

- decrease (increase) of crystal transparency and shift of absorption edge to the long (short) wavelength side;

- appearance (disappearance) of orange emission band at $\lambda_{m}=610 \mathrm{~nm}$;

- rise (vanishing) of photo-enhanced defect reaction resulting in photosensitivity degradation;

Any influence of electric field on the intensities of selfactivated emission bands at $\lambda_{m}=514 \mathrm{~nm}, \lambda_{m}=590 \mathrm{~nm}$ and $\lambda_{m}=715 \mathrm{~nm}$ was not observed.
Diffusion coefficients and activation energies of $\mathrm{Ag}$ diffusion parallel and perpendicular to the c- axis were determined. Diffusion anisotropy was found, diffusion being some times faster parallel to the $c$-axis.

\section{References}

1. F.A. Kröger, The chemistry of imperfect crystals, 654 p., Eds. North-Holland publishing company, Amsterdam (1964).

2. B.J. Boltaks, Diffuziya v poluprovodniakh, 462 p., Eds. Fizmatgiz (1961).

3. B.L.Timan, Yu.A. Zagoruiko // Fiz.Tverd.Tela, 21 (9), pp.2849-2851 (1979).

4. L.V. Borkovskaya, B.R. Dzhumaev, L.Yu. Khomenkova, N.E. Korsunskaya, I.V. Markevich // Proceedings of the $3^{d}$ Int. Conf. ASDAM-2000, Smolenice Castle, Slovakia, October 16-18, 2000, p. 187-190.

5. Physika soedinenii $A_{2} B_{6}$ edited by A.N. Georgobiani and M.K.Sheinkman, 320p., chap.4., Eds. Nauka, Moskva (1986).

6. Physics and Chemistry of II-VI Compounds, edited by M. Aven and J.S. Prener, 624 p., Eds. North-Holland publishing company, Amsterdam (1967).

7. I.B. Ermolovich, G.I. Matvievskaya, G.S. Pekar, M.K. Sheinkman // Ukr.Phys.Zhurn, 18 (5), pp. $729-737$ (1973).

8. I.B. Ermolovich, G.I. Matvievskaya, M.K.Sheinkman // Fiz. Tech. Poluprov., 9 (8), pp. 1620-1623 (1975).

9. H.H. Woodbury // J.Appl.Phys., 36 (7), pp. 2287-2293 (1965).

10. T. Lukaszewicz // Phys.Stat.Sol (a), 73 (2), pp. 611-616 (1982).

11. E.M. Zobov, G.G. Garyagduev, M.A. Rizahanov // Fiz. Tech. Poluprov., 21 (9), pp. 1637-1641 (1987).

12. G.A. Sullivan // Phys.Rev. 184(3), pp. 796-805 (1969).

13. E.D. Jones, H. Mykura // J.Phys.Chem.Sol., 39 (1), pp. 11-18 (1978).

14. E.D. Jones, H. Mykura // J.Phys.Chem.Sol., 41 (11), pp. 12611265 (1980)

15. L.V. Borkovskaya, B.R. Dzhumaev, L.Yu. Khomenkova, N.E. Korsunskaya, I.V. Markevich, M.K.Sheinkman // Semicond. Phys., Quant. Electron and Optoelectr., 3 (3), pp. 282$286(2000)$.

16. A.P. Batra, H.B. Huntington // Phys.Rev., 145 (2), pp. 542546 (1966). 